\title{
Round and round we go ...
}

As Schroder and Milano ${ }^{1}$ point out, there is quite a bit of hope surrounding the more recent left ventricular assist device (LVAD) trials. The 2 pumps that are mentioned are the HeartWare HVAD (Medtronic, Minneapolis, Minn) and HeartMate 3 (HM 3; Abbott, Chicago, Ill), with both promising a reduction in LVAD-related adverse events. Indeed, the excitement around these pumps are "palpable" and highlighted by the never before seen rate of enrollment with LVAD technology. Although the axial flow pump, HeartMate II (HM II; Abbott), historically has served as the workhorse for LVADs and been responsible for the proliferation of this technology, these 2 new centrifugal pumps have captured the imagination of the optimist. The attraction of a smaller pump that can be implanted either as entirely intrapericardial (HVAD) or intrathoracic (HM 3) cannot be denied, especially as more minimally invasive methods are being routine. In addition, eliminating the need for a more extensive surgical dissection is a significant benefit in the increasingly more complex patients who are being implanted with these pumps.

Aside from the benefit of a less surgically demanding operation, both pumps promise to improve patient outcomes and reduce the rate of pump thrombosis (PT). In fact, many speculate the rate of PT with the HM II has been the major hindrance to the dissemination of LVAD technology to the less-sick patient population. Although the Prevention of HeartMate II Pump Thrombosis Through Clinical Management (PREVENT) trial of standardized surgical and medical therapy has a reduction in PT rate of $2.9 \%$ at 3 months and $4.8 \%$ at 6 months with this pump, ${ }^{2}$ the results are still not acceptable.

It is encouraging that both the A Clinical Trial to Evaluate the HeartWare Ventricular Assist System (ENDURANCE; HVAD) and Multicenter Study of MagLev Technology in Patients Undergoing Mechanical Circulatory Support Therapy With HeartMate 3 (MOMENTUM 3; HM 3) trials have shown lower rates of PT compared with the HM II; oranges.

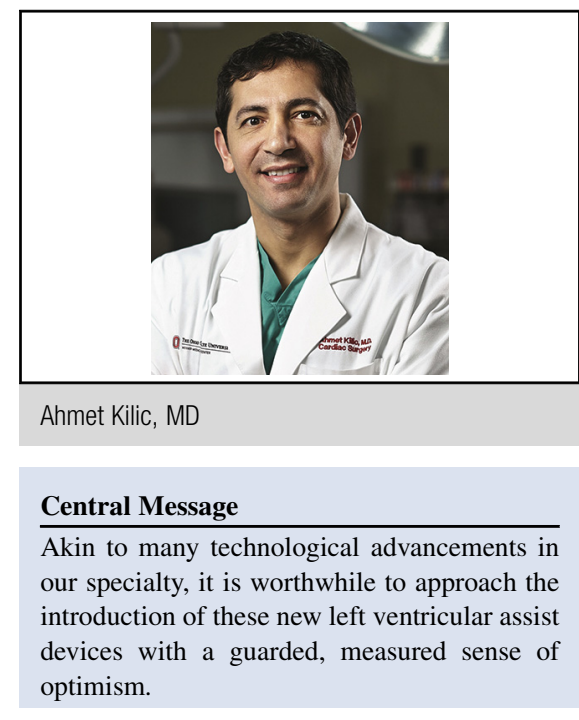

See Article page 850 .

however, these LVADs do require either a re-do sternotomy or re-do thoracotomy for device exchange versus the subcostal approach for the HM II. Although PT may be lower with these LVADs, a concern for both devices has been the risk of stroke. The ENDURANCE supplemental device presented earlier this year has shown reduction in strokes with close blood pressure management but only compared with the historic HVAD patients in the original ENDURANCE trial. ${ }^{3}$ Likewise, the MOMENTUM III trial still in infancy has shown no evidence of pump thrombosis in the original cohort at 6 months but once again with an accompanying rate of strokes. ${ }^{4}$ A significant fact to remember for the MOMENTUM III trial is the strict inclusion criteria selecting for perhaps healthier patients and bringing up the question of comparing apples with

Akin to many technological advancements in our surgical specialty, it is worthwhile to approach both pumps with a guarded, measured sense of optimism. The ability to offer newer LVADs in a more minimally invasive method with less surgical trauma is a valuable tool in the armamentarium of surgeons treating advanced heart failure. However, the ability to anticipate and avoid complications from our interventions is a central tenant of surgeons, and we must curb our enthusiasm as to not trade a known set of problems for other, unanticipated adverse events. There will always be the next generation of LVAD touted as the next great savior, but blind enthusiasm must be weighed against our oaths to first do no harm. Rather than the traditional debate 
of which pump is best, we should fit our technology with the specific needs of each patient. By learning from each iteration of technology, we will be able to offer our otherwise-moribund patients not only survival but sustainable adverse-free quality of life.

\section{References}

1. Schroder JN, Milano CA. A tale of two centrifugal left ventricular assist devices. J Thorac Cardiovasc Surg. 2017;154:850-2.
2. Maltais S, Kilic A, Nathan S, Keebler M, Emani S, Ransom J, et al. PREVENTion of HeartMate II pump thrombosis through clinical management: the PREVENT multi-center study. J Heart Lung Transplant. 2017:36:1-12.

3. Milano CA, Rogers JG, Tatooles AJ, Bhat G, Slaughter MS, Birks EJ, et al. The treatment of patients with advanced heart failure ineligible for cardiac transplantation with the HeartWare Ventricular Assist Device: results of the ENDURANCE Supplement Trial. J Heart Lung Transplant. 2017;36: S10.

4. Mehra MR, Naka Y, Uriel N, Goldstein DJ, Cleveland JC Jr, Colombo PC, et al. A fully magnetically levitated circulatory pump for advanced heart failure. N Engl J Med. 2017;376:440-50. 